\title{
C2 - command and control : un système de systèmes pour accompagner la complexité
}

\section{Bernard Claverie et Gilles Desclaux}

\section{revues.org}

Édition électronique

URL : http://

communicationorganisation.revues.org/5449

DOI : $10.4000 /$

communicationorganisation.5449

ISBN : 979-10-300-0127-3

ISSN : 1775-3546

\author{
Éditeur \\ Presses universitaires de Bordeaux \\ Édition imprimée \\ Date de publication : 1 décembre 2016 \\ Pagination : 255-278 \\ ISBN : 979-10-300-0069-6 \\ ISSN : 1168-5549
}

Distribution électronique Cairn

\section{CAIRN INFO}

CHERCHER, REPÉRER, AVANCER.

Référence électronique

Bernard Claverie et Gilles Desclaux, « C2 - command and control : un système de systèmes pour accompagner la complexité », Communication et organisation [En ligne], 50 | 2016, mis en ligne le 01 décembre 2019, consulté le 10 février 2017. URL : http://communicationorganisation.revues.org/5449 ; DOI : 10.4000/communicationorganisation.5449 


\title{
C2 - command and control: un système de systèmes pour accompagner la complexité
}

\author{
Bernard Cloverie' et Gilles Desclaux ${ }^{2}$
}

\section{Introduction}

La gestion moderne des opérations militaires s'appuie sur une conception du commandement et du contrôle $(\mathrm{C} 2)$ totalement imprégnée des dimensions nouvelles des technologies de l'information et de la communication. Cet envahissement de la technicité a donné lieu, dans les années 1980, à une évolution de la désignation du système comme C3I, en adjoignant au sigle les dimensions de la communication et du renseignement (intelligence). $\mathrm{Au}$ tournant du siècle, la technologie s'est explicitement invitée en faisant évoluer le C2 en C4ISR, ou aujourd'hui même en C4ISR-TAR, pour Command, Control, Communications, Computers, Intelligence, Surveillance, Reconnaissance, et Target Acquisition et Reconnaissance spécifique (acquisition de cibles) (C4ISR Network, 2015).

Le C4ISR-TAR est le sigle utilisé notamment par le Département de la Défense américain (DoD) pour désigner l'ensemble des moyens et processus militaires organisés et structurés en vue de la conduite des opérations, de leur commandement et de leur contrôle. Ce mouvement technologique a fait entrer le $\mathrm{C} 2$ dans une définition centrée sur l'utilisation des moyens.

Parallèlement, et comme le soulignent certains auteurs (Alberts et Hayes, 2006), le domaine s'est constitué de manière réglementaire, à partir de définitions institutionnelles et légales revêtant une dimension managériale à valeur juridique. Ainsi en est-il des textes du DoD ou de l'OTAN. Pour le « DoD Dictionary of Military and Associated Terms ", le C2 correspond

1 Bernard Claverie est professeur des universités, directeur de l'École Nationale Supérieure de Cognitique (Bordeaux INP). Il effectue ses recherches au sein de l'UMR CNRS 5218 et du Human Engineering for Aerospace Lab. (laboratoire commun ENSC-Thales).

2 Gilles Desclaux est général de corps aérien (2s), président de RACAM (Réunion Aviation Civile et Aviation Militaire). Il a été le commandant du CDAOA (Centre de la Défense Aérienne et des Opérations Aériennes) et expert C2 à l'OTAN. Il est directeur extérieur de Thales Raytheon Systems et consultant pour des industries et groupes de communication. 
à « l'exercice de l'autorité et de la direction de moyens assumés par le commandeur de forces militaires qui lui sont attachées pour la réalisation de sa mission » (Jcosd 2008) .

Le terme de " commandeur » doit être précisé. En France, il se réfère plus à la traduction littérale du terme anglo-saxon " commander " qu'à un grade établi dans la hiérarchie civile ou militaire francophone. Il s'agit ici d'une position hiérarchique fonctionnelle, plus ou moins longue et reconnue de tous les acteurs à un moment donné. Elle peut d'ailleurs s'inscrire dans une constante de temps très brève, de quelques heures à quelques jours ${ }^{4}$ ou revêtir une durée plus longue allant de plusieurs mois à quelques années; elle est rarement définitive pour une personne, sauf cas particuliers rencontrés en matière monarchique ou religieuse. La définition précise que le $\mathrm{C} 2$ est assumé " grâce à un ensemble organisé de personnels, d'équipements, de communications, de dispositifs et de procédures qui sont employés par le commandeur pour la planification, la direction, la coordination et le contrôle des forces et des opérations dans l'accomplissement de sa mission ».

Cette notion de "Commander » est fortement associée celle de stratégie. La stratégie est fondamentalement ce qui transforme, ce qui fait passer d'un état à un autre. Le commandeur s'appuie sur un $\mathrm{C} 2$ pour atteindre un état final recherché qui est un changement d'état.

La stratégie n'est ni une méthode, ni un plan, c'est plutôt un acte de détermination. (Commander's Intent). Elle est avant tout portée par un homme ou une femme et le $\mathrm{C} 2$ est son multiplicateur de force qui lui permet de faire émerger la bonne stratégie et de l'appliquer avec performance: mieux comprendre, mieux décider de l'application de la force, minimiser les risques et mieux saisir les opportunités.

Lorsqu'un commandeur déclare qu'il a la supériorité stratégique, il dit qu'il peut décider du lieu, du moment de l'affrontement et même de l'adversaire. Le dispositif C2 lui permet alors d'exercer le pouvoir (capacité à agir dans l'instant) et la puissance (capacité projetée de manière potentielle vers l'avant). C'est grâce à la performance de ce dispositif, qu'il peut décider plus vite que l'adversaire ou le concurrent, et conserver l'initiative.

Le «NATO glossary » reprend cette définition. Le C2 est « l'ensemble des fonctions des commandeurs, de leurs équipes et autres corps de commandement pour le maintien des forces, la préparation des opérations, et la direction des troupes en vue de leurs missions ». La notion englobe diverses dimensions que précise le glossaire. En premier lieu celle de l'information, avec « son acquisition continue, sa fusion, son examen et sa représentation, ainsi que son analyse permettant l'évaluation et la conscience de la situation ».

\footnotetext{
3 « The exercise of authority and direction by a properly designated commander over assigned and attached forces in the accomplishment of his mission. Also called C2. » (Online Department of Defence Dictionary of Military and Associated Terms).

4 On parle alors plus de « Chef de mission » ou « On Scene Commander »: OSC.
} 
S'y ajoutent « la planification et la constitution du projet de commandement (plan), la répartition des moyens et des tâches aux différents éléments des forces (tasking), l'opérationnalisation, l'organisation et le maintien de la coopération de ces forces et toutes les formes de leur soutien $»^{5}$. La définition illustre une série d'actions qui donnent au $\mathrm{C} 2$ une dimension d'information, d'analyse, de décision, de logistique et de mise en œuvre de moyens et de leur soutien pour répondre à une situation à maintenir ou faire évoluer (Desclaux 2006).

Pour Alberts et Hayes (op. cit. 2006), les études sur le C2 doivent s'affranchir de ces définitions réglementaires ou techniques pour aborder une dimension explicitement scientifique. Ainsi, la théorisation portant sur l'ensemble du C2 s'est-elle développée, bien entendu à l'initiative du DoD mais également des pays de l'OTAN et de grands industriels fournisseurs des moyens technologiques concernés. C'est autour de plusieurs groupes de réflexion et d'études qu'une dimension générique a été développée, constituant un champ d'étude théorique spécifique du «command and control ».

Pourquoi ce domaine théorique se distingue-t-il du volet purement technologique de ces dispositifs ou réglementaire des méthodes de gestion et management? En fait, les systèmes modernes concernés sont extrêmement compliqués et leur usage complexe. Ils impliquent de nombreux éléments technologiques et humains en interaction: hommes-hommes, hommetechnologie, technologies-technologies. Ces sources de complexité adressent l'ensemble des processus et interactions sur plusieurs niveaux et entre ces niveaux. C'est cette dimension anthropotechnique qui est à la source principale de la complexité, de l'incertitude et de l'incomplétude des modèles de compréhension et donc de difficulté de maîtrise du C2.

Cet article aborde successivement, après une description du C2, la dimension de complexité et celle de la multidimensionnalité des espaces théoriques de la mise en ouvre et des espaces d'application du « command and control».

\section{Domaines d'application du C2}

$\mathrm{Si}$ le $\mathrm{C} 2$ est une dimension première de l'organisation militaire (Boyes et Andriole, 1987) de l'ensemble des différentes forces et de leur interopérabilité (Joergensen et al. 2005; Larsen 2006), y compris pour les coalitions, il concerne également d'autres domaines d'application moins martiaux. Le C2 est notamment utilisé dans des secteurs habituellement préoccupés par la gestion des crises. Ainsi en est-il des organismes en charge de la sécurité publique, de ceux gérant les transports et les dynamiques de véhicules, de

\footnotetext{
5 NATO glossary : $\mathrm{C} 2$ is « the functions of commanders, staffs, and other Command and Control bodies in maintaining the combat readiness of their forces, preparing operations, and directing troops in the performance of their tasks. The concept embraces the continuous acquisition, fusion, review, representation, analysis and assessment of information on the situation; issuing the commander's plan; tasking of forces; operational planning; organizing and maintaining cooperation by all forces and all forms of support. »
} 
fluides ou d'énergie pouvant faire l'objet de dysfonctionnements, de ceux en charge de la sécurité environnementale et de la lutte contre les pollutions, les ravages liés aux parasites végétaux ou l'expansion des épidémies et épizooties, etc. D'un autre côté, les entreprises ou organismes gérant des programmes complexes nécessitent souvent un contrôle strict de l'erreur ou la gestion des écarts à la trajectoire pour un objectif précis. C'est le cas des programmes spatiaux et de tous les dispositifs de construction ou de réalisation nécessitant une précision anticipatoire stricte, avec un contrôle immédiat des écarts avec anticipation des conséquences. Ces dimensions, gestion de crises, maîtrise de l'erreur et anticipation des conséquences, sont des caractéristiques des domaines utilisant le $\mathrm{C} 2$.

Dans son sens le plus large, le C2 est donc à la fois une méthode et un procédé associés à une activité complexe nécessitant totalement ou partiellement un contrôle strict. Celui-ci est défini par un "Operational Design» qui doit conduire à l'effet final recherché, et la synchronisation des actions est indispensable. La décision peut, quant à elle, être partagée jusqu'aux plus bas échelons si nécessaire dans une structure privilégiant l'agilité et la délégation dans le cadre défini. La Défense n'en est alors qu'un cas d'application particulier. Néanmoins, les principes du C2 sont issus de l'organisation militaire, et on note d'ailleurs son influence dans ces différents autres domaines d'application. La pervasion des méthodes est évidente, de même qu'un vocabulaire martial passe métaphoriquement dans le domaine civil (Wee et al. 1991): campagne commerciale, guerre des prix, état-major, marketing warfare, war-room, stratégie de conquête, manœuvres, etc. et plus traditionnellement, délégué général, capitaine d'industrie, etc. (Le Roy 1997).

De manière générale, on constate que les méthodes du C2 peuvent être appliquées dans une mesure plus ou moins importante aux systèmes civils institutionnels et commerciaux. Les éléments de la complexité du système y sont abordés selon les mêmes principes. Le $\mathrm{C} 2$ permet alors d'envisager et de structurer des démarches organisées vers un but défini par le commandement, contrôlées par un dispositif humain informé par les différents apports statistiques, tableaux de bord et indicateurs stratégiques, et augmentées par un ensemble technologique d'information, de communication et d'aide à la décision.

\section{Commandement et contrôle}

En sciences humaines, le commandement est la capacité qu'a un individu ou un groupe d'acquérir ou d'exercer de l'autorité sur un ou plusieurs individus ou groupes en fonction de critères reconnus par ces derniers. $\mathrm{Au}$ premier plan s'impose la compétence, mais également la popularité ou la hiérarchie s'inscrivant dans un processus de leadership. Dans les domaines 
institutionnels, notamment administratif et militaire, le commandement désigne par métonymie l'autorité elle-même ou souvent le siège de cette autorité.

Le contrôle, quant à lui, représente l'aptitude à diriger un système et à le maintenir dans un état permettant une action concrète et définie. La notion s'inscrit directement dans le domaine cybernétique. Il vise notamment à maîtriser, voire supprimer le changement, et organiser le système en combattant l'entropie. Il se réfère à trois dimensions complémentaires. La première est informative, donnant des valeurs de mesure de l'état du système contrôlé, ou des éléments du système, permettant de vérifier s'ils inscrivent leur évolution temporelle dans un espace de variation déterminé. La seconde dimension est préventive, anticipant les possibilités de dérive et empêchant que ne se produisent des écarts à l'attente ou ne correspondant pas à l'objectif souhaité. Cette seconde dimension peut être considérée comme caractérisant le volet négatif du contrôle. La troisième est incitative, de caractère positif, en promouvant les possibilités voulues, et en favorisant leur mise en œuvre, leur développement et leur expression en fonction des objectifs commandés. Le contrôle a notamment pour but de réduire l'incertitude dans le système. Il devient alors une des principales sources de stress des organisations humaines, par peur ou angoisse de perte de contrôle ou de dépassement des capacités de retour à l'état espéré. La " psychologie du contrôle » est un exemple du rôle de tels déficits dans l'apparition de l'angoisse et de sa gestion par des processus de contrôle.

Pourquoi aborder le $\mathrm{C} 2$ selon cette perspective ? La cybernétique est une science qui traite d'une part de l'identification, de l'analyse, de la modélisation, et d'autre part de la commande et du contrôle des systèmes dynamiques. Ses fondements théoriques s'inscrivent dans les mathématiques, la théorie du signal, les technologies de l'information et de la communication. Elle utilise des outils tels que la logique et l'informatique théorique et permets, grâce à elles, d'expérimenter les effets de leur implémentation sur des cas concrets. D'une part elle aborde concrètement le fonctionnement des systèmes, d'autre part elle propose une démarche d'ordre abstrait. Celle-ci s'organise autour de la théorisation de la structure logique des fonctionnements couplés systèmeenvironnement et de leur modélisation mathématique selon les règles de la logique. On parle ici de «logique des automates».

Ainsi dotée d'une théorie propre, elle se distingue alors de la théorie de l'information, qui construit une définition quantitative et objective de la notion d'information, et des théories de la communication qui étudient les indices quantitatifs ou qualitatifs des relations entre des agents (machines, êtres, milieu). On pourra dire que les aspects déterministes du C4ISR sont étudiés par la théorie de l'information, notamment en ce qui concerne les points centraux de la transformation de l'information et son codage, du transport et du transfert, du filtrage et du stockage, et plus en aval, de la valeur 
et de la signification de l'information à chaque niveau de son traitement. La commande n'est possible que si elle s'appuie sur le contrôle. La communication est le moyen de transport des données d'un endroit à un autre, d'un niveau à un autre, de l'environnement à l'ensemble du système et des éléments du système à l'extérieur. L'intelligence est le produit résultant de la collecte, de l'analyse, de l'évaluation et de l'interprétation des données pertinentes pour atteindre le résultat souhaité. Ces données sont recueillies par les capteurs, transformées en informations par des moyens essentiellement technologiques et enfin converties en connaissances par les compétences cognitives du commandant et de ses analystes.

Ce volet applicatif est du domaine des $\mathrm{STIC}^{6}$. Il permet de commander et de contrôler un système en respectant un plan ou un cahier des charges de son évolution temporelle. On évoque alors le concept de pilotage qui a d'ailleurs donné son nom à la cybernétique (Claverie et al. 2014). La cybernétique est donc un art applicable à la théorisation du $\mathrm{C} 2$ et le $\mathrm{C} 2$ une forme de réification de la cybernétique. Certains auteurs proposent même " une fusion des deux approches » (Builder et al. 1999). Pour ce qui est de la conceptualisation du C2, centrée de manière plus abstraite sur la compréhension, la conception et la formalisation des systèmes d'action et de régulation, on parle de "systèmes formels » dont la qualité est l'indépendance des supports réels. Qu'il se réfère au monde artificiel ou naturel, abstrait ou concret, le système formel est un être théorique qui peut recevoir une déclinaison conceptuelle. Il transforme une représentation abstraite d'entrée, qui peut être d'essence purement mathématique, d'un signal ou d'une série de signaux donnés, en une représentation de sortie, tout en itérant ses transformations à chaque niveau par des boucles de rétroactions couplées avec l'environnement ou entre différentes unités d'un calcul opéré par un ou des programmes internes. C'est donc un « être computationnel » dont les transformations et celles du milieu relèvent de la théorie des algorithmes (Claverie et Desclaux 2015).

\section{C2, conflits et modernité}

Dans les batailles traditionnelles, le commandeur est habituellement entouré de son groupe de commandement, dans une situation élevée d'observation du champ de bataille, et avec une disposition immédiate d'un système d'estafettes permettant à chaque commandant sur le terrain de recevoir des ordres d'action ou de modulation de l'action de ses troupes. Pour plus de précision, les bleus se battent contre les rouges (ou toutes autres couleurs nécessaires à leur distinction) aux sons de leurs trompettes et tambours. Ainsi le commandeur utilise ses propres yeux et oreilles pour recueillir les données, mobilise ses compétences cognitives pour traiter les données et évaluer les flux et reflux des troupes en bataille, évalue les choix qui s'offrent à lui pour définir les actions

6 STIC: Sciences et techniques de l'Information et de la Communication. 
les plus adéquates à l'objectif, met en œuvre sa décision par des messages et, si nécessaire, répète le processus par itérations successives jusqu'à la réalisation de cet objectif (Cooper 1994) qui est le plus souvent d'attrition de l'ennemi. Dans un tel processus, le commandement est évident et le contrôle se résume à une suite itérative d'ordres de régulation à destination des commandants des forces engagées.

Les opérations militaires modernes sont fondamentalement différentes, le commandement, le contrôle des forces et la conduite des opérations sont d'une autre espèce, et l'objectif est plus d'influence que de force (Warden 1995).

Les conflits modernes ont changé complètement l'équation du commandement et du contrôle dans la conduite des opérations militaires. La Première Guerre mondiale a mis en exergue l'importance d'une série de dimension jusqu'ici négligée ou considérée comme secondaires. La technologie a tant progressé quelle tend à changer tous les modes d'affrontement, renvoyant le conventionnel au passé et le futur à l'incertitude. L'invisibilité s'est substituée au camouflage, lui-même généralisé comme la forme moderne des couleurs de la guerre, alors qu'elle s'imposait également comme arme offensive avec l'apparition des gaz et d'autres terribles moyens qu'aujourd'hui les nations s'entendent pour en interdire et l'usage et même la détention. Les appareils de surveillance aérienne se sont armés et influent alors sur le déroulement des opérations terrestres, alors que les discussions sont aujourd'hui encore vives entre les partisans et les détracteurs des robots et drones tueurs. Enfin, l'information, son accès, sa diffusion et son chiffrement sont devenus des préoccupations majeures avec les méthodes de cryptographie qui ont tant fait avancer la recherche mathématique et la sûreté des opérations économiques. La Seconde Guerre mondiale puis les conflits de décolonisation ont poussé l'équation encore plus loin, avec l'apparition de la cybernétique de Wiener, Rosenblueth, Bigelow, McCulloch ou Pitts, et en France Couffignal (1963) et de l'informatique avec Turing, Von Neumann et leurs successeurs, avec en Europe Steinbuch (1957) ou Dreyfus qui fut à l'origine du mot en français en 1963 (voir Claverie, 2005). L'information est devenue centrale, qu'elle soit considérée à son recueil, son analyse, son transfert, sa distribution et sa compréhension par les hommes. C'est à cette dernière étape que s'est constituée plus récemment de l'ingénierie du couplage homme-système avec les spécialistes qui s'inspirèrent directement des deux mouvements précédents : cybernétique et informatique.

Comme l'écrivait Moshe Dayan (1966) : « Où sont les bons vieux jours de guerre simple lorsque, quand l'heure du combat approchait, le commandant montait sur son cheval blanc, et qu'au son de la trompette il fonçait vers l'ennemi ... en criant "Suivez-moi !" ? ». Les actions militaires sont devenues technologiques; ce sont celles des capteurs, de l'information, de l'intelligence et de la décision efficace en situation d'incertitude pour une action elle-même 
empreinte de la soumission à l'inattendu et de l'adaptation de l'action, pour agir souvent à distance par des actions de vecteurs humains ou automatiques qui s'autorégulent eux-mêmes selon la logique des réseaux.

Dans un tel contexte, le commandement correspond à la mobilisation des moyens matériels et humains disponibles d'une manière qui permet d'atteindre un résultat souhaité. Ce résultat correspond à une modification sensible de l'état de l'environnement. Le contrôle consiste quant à lui à observer et mesurer la situation environnementale actuelle afin d'évaluer la différence qui sépare le système de l'état souhaité, et à émettre des directives continuellement ajustées qui maintiendront les évolutions de l'état actuel vers le résultat souhaité, ou le maintien de cet état une fois atteint. En termes de conflit, de surveillance ou de maintien de la paix, le $\mathrm{C} 2$ s'attache à un résultat qui ne se limite pas au domaine militaire. L'engagement est souvent même secondaire, mobilisé comme moyen de régulation ou d'évolution, avec des logiques d'information, d'influence, de «soft power» et de frappes chirurgicales?

\section{Le C2 comme système cybernétique}

Pour comprendre facilement le C2, il faut revenir aux principes d'un système cybernétique simple, puis le complexifier progressivement (Claverie, Desclaux 2015, op. cit.). Un exemple de modèle formel élémentaire de régulation des systèmes est celui du thermostat : il s'agit d'une machine aujourd'hui numérique, dotée de capteurs et d'un dispositif de réglage (la commande) permettant d'ajuster la température d'un milieu par l'intermédiaire d'un système de régulation (le contrôle) qui actionne un système de température (le comportement). Le chauffage correspond à un premier comportement et la réfrigération à un second. L'utilisateur du dispositif (le commandeur) impose sa décision (ordre) pour atteindre grâce au dispositif un effet désiré (but). C'est l'évolution vers cet effet qui, en retour et grâce aux informations des capteurs, déclenche un comportement choisi dans une gamme finie de variétés. Un ensemble de relations permet de communiquer de l'étage de commande à celui du contrôle, puis à celui du comportement, et de provoquer un effet sur l'environnement. Chaque étage est doté d'une fenêtre de possibilités (degré de liberté) fixée par le programme. On remarque déjà que, pour ce système cybernétique des plus simples, deux caractéristiques sont incontournables: l'articulation du commandement et du contrôle pour l'action selon la décision prise, et la distribution de l'information généralisée.

Dans l'automate formel, le commandeur décide et le contrôleur à une certaine liberté de choix (algorithme) dans une gamme fixée d'ordres donnés à l'effecteur qui agit alors directement sur le milieu. À chaque instant, le contrôleur informe le commandeur, l'effecteur informe le contrôleur et le

7 « Contrary to Clausewitz, destruction of the enemy military is not the essence of war; the essence of war is convincing the enemy to accept your position, and fighting his military forces is at best a means to an end and at worst a total waste of time and energy. » (Warden 1995). 
commandeur. Chacun est de plus directement informé de l'état général du système à partir d'un dispositif de capteurs (information situationnelle) et de boucles de rétroaction. L'information contenue dans le système correspond à l'ensemble des signaux " commande-contrôle-comportement-effets " auquel est associé l'ensemble des « feedbacks » (voir détails à la figure 1).

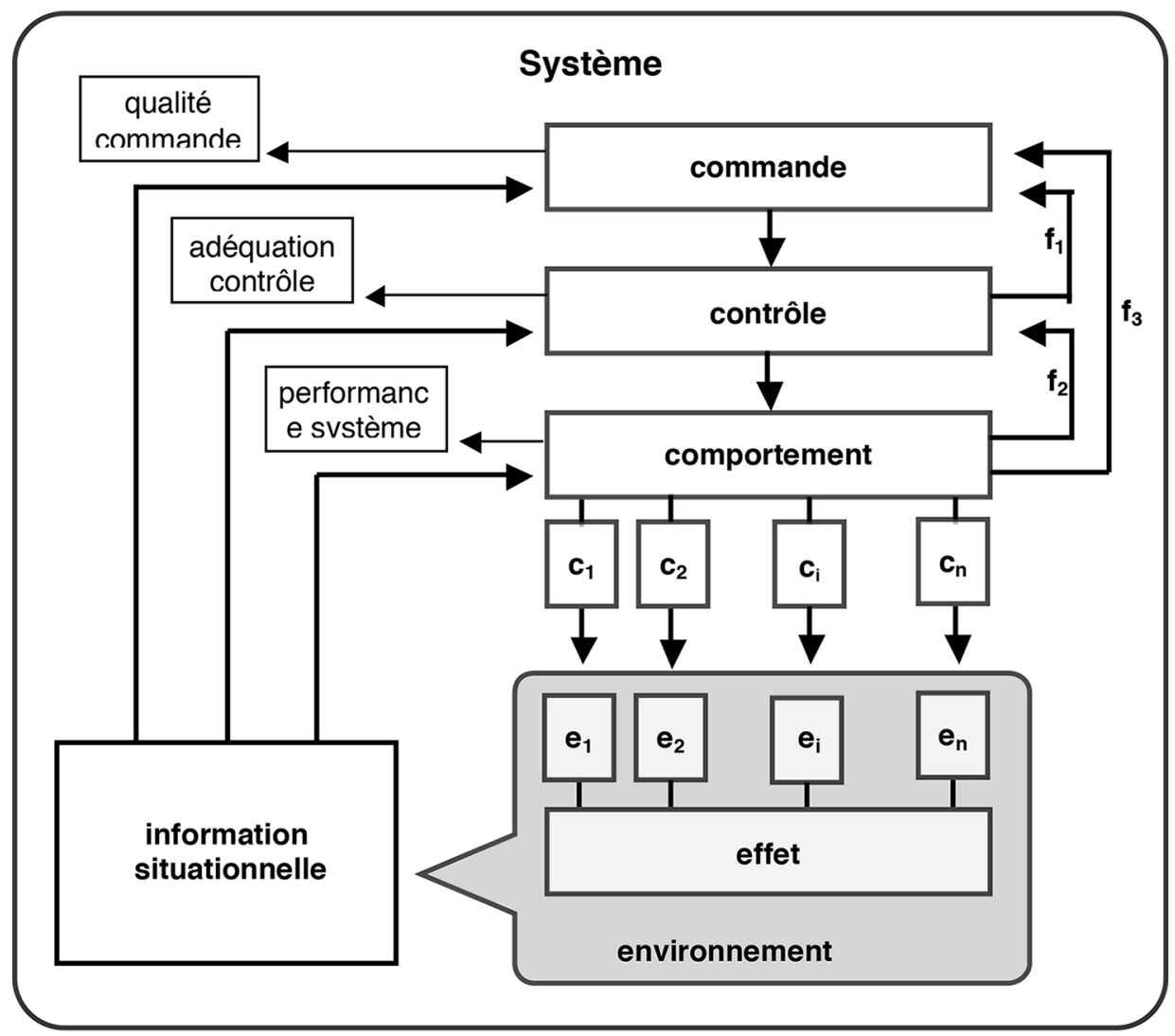

Figure 1 : Modèle conceptuel « Commande-Contrôle-Comportement » et « feedback ». Inspiré d'Alberts \& Hayes (2005)

Chaque système est donc défini selon des règles définissant un modèle conceptuel de base: commande et contrôle, comportement et feedback. Ce modèle a fait l'objet de multiples instanciations, que ce soit pour les machines mécaniques, les machines énergétiques ou les machines informationnelles. C'est surtout dans le domaine de ces dernières que l'application est la plus spectaculaire avec le développement des ordinateurs mais également celui des multiples dispositifs électroniques modernes. Les ordinateurs peuvent se connecter entre eux, et les opérateurs deviennent autant d'éléments de réseaux opérationnels interagissant dans le cadre d'un projet global défini 
pour le système entier. Ce système et ses sous-systèmes, les capteurs, senseurs, transmetteurs, filtres, amplificateurs, analyseurs, afficheurs, etc. participent à la production quasi sans limite de données nouvelles dont l'exploitation est impossible sans d'immenses puissances de calcul. Quels que soient les dispositifs informationnels collaboratifs, ils deviennent tous aujourd'hui producteurs de données à la fois pour leur propre régulation, mais également pour enrichir des bases de données spécialisées ou généralistes, dans un monde de « big data » (C4ISR \& Networks 2015), contribuant à une nouvelle forme de « complexité computationnelle » du calcul et de l'information (Cooper 1994).

Tous ces dispositifs interconnectés comportent eux-mêmes des soussystèmes constitués sur un modèle similaire. Ils sont à plusieurs niveaux les éléments de super-systèmes devenant des "systèmes de systèmes »? Ceux-là font depuis quelques années l'objet d'une "ingénierie des systèmes " que certains nomment « ingénierie de la complexité » (Le Moigne 1999; Luzeaux et al. 2011). Dans une telle organisation modulaire, des modules comportent d'autres modules de même structure, répondant pour cela aux caractéristiques d'une autre forme de complexité dite "dimensionnelle " (ibid.). Chaque module et chaque étage s'attachent à maximiser les caractéristiques de qualité de commande, l'adéquation du contrôle, la performance système, la pertinence d'information, et la précision de la mission. Le système global illustre ici la définition d'un "système artificiel complexe » (Simon 1996) sur la base des principes de la cybernétique.

\section{Le $\mathbf{C 2}$ en quelques questions ...}

Le volet opératoire et instrumental du C2 (C4ISR-TAR) est maintenant bien connu, développé et formalisé efficacement dans de nombreux manuels, notes et circulaires. On constate pourtant que le volet théorique du C2 ne permet pas encore d'en promouvoir une conception simple et claire. Des centres, des laboratoires et de réseaux de recherche se consacrent depuis quelques années au domaine. Un vaste corpus de connaissances est ainsi aujourd'hui accessible. Il n'y a pourtant pas de théorie unifiée du C2.

Plusieurs tentatives ont eu lieu, débouchant sur des résultats plus ou moins convaincants (Coakley 1991). Au bénéfice des avancées, il faut citer les travaux du CCRP (Command and control research program) du DoD avec notamment ceux d'Alberts et Hayes (2004 op. cit. 2006). Le programme expérimental ELICIT étudie quant à lui en situation simulée différents paramètres du C2 (2006) et est aujourd'hui utilisé pour la simulation et la formation (Tossel et al. 2008 ; Rudy 2011). D'autres initiatives sont à signaler, notamment avec des entreprises. Le laboratoire de simulation EXC3ITE (Experimental command, control, communications and intelligence technology environment) en est un exemple (Yates et al. 1999). Les activités du Centre d'analyse et de simulation pour la préparation des opérations aérienne (CASPOA) à Lyon, et celles du 
Centre d'excellence pour le soutien du commandement et du contrôle (C2$\mathrm{CoE}$ ) implanté à Ede aux Pays-Bas, tous deux centres de référence OTAN, s'inscrivent dans ce principe. Ils utilisent la simulation pour la formation au C2 des personnels de l'Armée de l'Air ou des spécialistes de l'interopérabilité. Ce type de pédagogie nécessite des moyens matériels et humains importants et un investissement significatif des individus afin de développer une maîtrise, faute d'une compréhension exhaustive, de la complexité des systèmes de commandement et conduite des opérations (niveaux d'opérateur, de contrôleur ou coordinateur, et de commandeur).

$\mathrm{Au}$-delà de la recherche appliquée telle que le permettent les dispositifs précédents dans le domaine direct des applications civiles ou militaires, le défi de la recherche est de développer un ensemble de principes fondamentaux qui peuvent constituer la base d'une théorie du commandement et du contrôle.

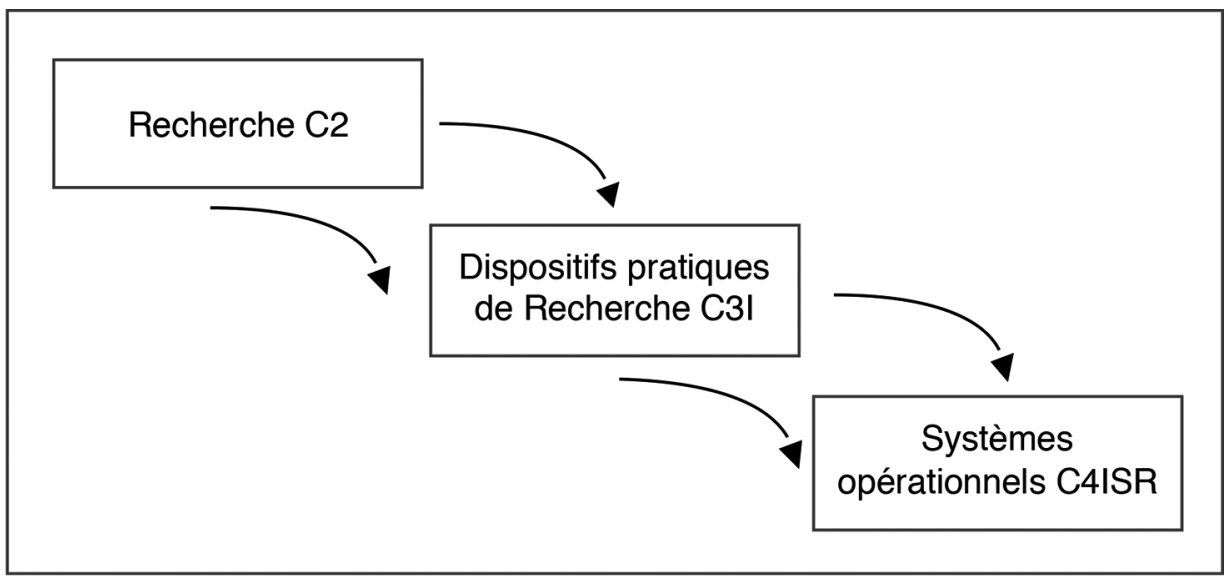

Figure 2 : Dynamique de la recherche en C2 (inspiré de Yates et al., 1999)

La difficulté de l'entreprise réside dans la complexité du C2 (Cooper op. cit. ; Olmedo 2010 ; Woodcock 1987). Sa rationalisation s'accorde mal des décompositions instrumentales, et l'on peut dans un premier temps différencier deux dimensions. La première est ascendante (Taylor et Snell 1988), en termes de nombre de niveaux et d'étapes nécessaires à l'obtention d'une solution, dans laquelle le $\mathrm{C} 2$ est organisé à partir de l'assemblage des pièces d'un « jeu de construction ", sorte de Lego du C4ISR-TAR. Cette approche se heurte rapidement à la barrière de l'explosion exponentielle de l'information gérée et crée, notamment par l'inclusion de la variabilité attachée à chaque brique et à ses différentes possibilités d'articulation aux autres. Le facteur humain joue un rôle central dans cette variabilité et les caractéristiques individuelles des contributeurs au système (démarche constructive ou bottom-up) ainsi que la nature de leurs interrelations participent à une expansion combinatoire. La 
démarche consistant à analyser le système de manière descendante (Levis et Athans 1988), c'est-à-dire en décomposant les éléments et en définissant les relations entre les éléments, constitue une seconde approche qui rencontre le principe d'incertitude due à la sensibilité aux conditions initiales. Elle se retrouve confinée dans des descriptions statistiques (démarche déductive ou top-down), parfois approximatives, toujours réductrices, et empreinte d'imprévisibilité.

\section{Les niveaux de complexité du C2}

On peut déterminer, dans ces mouvements d'analyse, au moins trois niveaux de complexité : la complexité d'incertitude, la complexité dimensionnelle, la complexité computationnelle (Cooper op. cit. 1994).

La complexité due à l'incertitude de l'information est, pour des auteurs comme Levis et Athans (op. cit. 1988) le " grand défi » du C2. Rappelons le théoricien de la guerre moderne, Clausewitz (1832), dont on connaît l'influence au-delà du domaine strictement militaire, en sciences humaines, politiques et économiques, et selon lequel «Une grande partie de l'information obtenue en temps de guerre est contradictoire, une partie encore plus grande est fausse, et la plus grande partie est de loin passablement douteuse ». Ces trois caractères d'incertitude sont eux-mêmes relatifs à des dimensions d'incertitude technologique, d'incertitude de la situation et d'incertitude $\mathrm{du}$ facteur humain. Le $\mathrm{C} 2$ va s'attacher à minimiser ces incertitudes et tendre à ramener la situation à un état de certitude relative sans laquelle le commandement et le contrôle sont difficiles à mettre en œuvre (cf. figure 3 - passage de la situation A à la situation B).

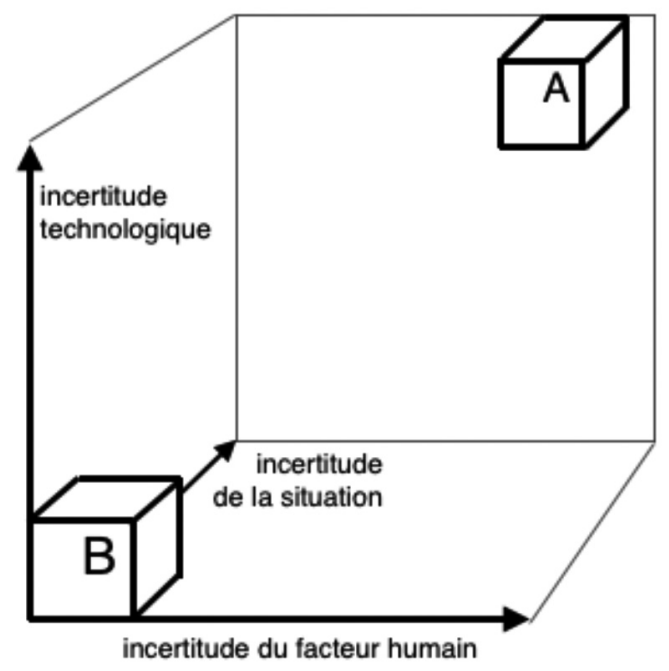

Figure 3 : Réduction d'incertitude dans l'espace de complexité de Clausewitz : passage de la situation A à la situation B. 
La maintenance des appareils de recueil et leur performance partielle, la mise à jour des moyens d'analyse, la fusion et les approximations mathématiques, la limite de calcul d'ordinateurs arrondissant leurs résultats ${ }^{8}$, la nécessité de réduction pour une représentation imagée souvent immédiate à destination d'opérateurs eux-mêmes parfois diminués, cognitivement saturés ou psychologiquement stressés qui communiquent parfois imparfaitement, sont autant d'exemples d'une longue liste de l'appauvrissement et de la relativité de l'information. Si ce type d'incertitude peut être réduit par l'évolution de la technologie, l'amélioration des algorithmes de calcul, et le développement de procédures d'ingénierie cognitive plus élaborées et plus sûres, on est constamment confronté d'une part à une augmentation des équipements et de leur puissance, mais également à une évolution des contre-mesures et de la furtivité des sources d'information.

Une source importante d'incertitude est due aux activités humaines extérieures. Les actions volontaires de parasitage, et de saturation des senseurs, les cyberattaques ou les actions physiques directes de dégradation des capteurs, des réseaux ou des centres de décision et contrôle en sont des exemples. L'ennemi est souvent intelligent, actif et imprévisible. Il dispose d'un vaste répertoire de techniques ayant pour vocation la production d'incertitude, et tous les composants du système sont susceptibles d'en être victimes. Un des buts du concepteur du système sera la robustesse des éléments et réseaux, ceux de l'utilisateur privilégieront celle des procédures, le maintien en condition opérationnelle des dispositifs et leur protection face aux attaques physiques ou informationnelles. Tel est le ça par exemple de la tendance à la suppression de la communication par la voix et sa substitution par chats et forums sécurisés utilisant des liaisons de grande vitesse et robustesse (e.g.: liaisons 16, 22 et J-over-IP) (Kometer 2007). Le renseignement (Intelligence) entre dans ce domaine en permettant de recouper les sources et de minimiser l'incertitude tout en donnant des informations qui échappent à certaines technologies traditionnelles.

La principale source d'incertitude humaine est paradoxalement interne ; elle est relative aux faiblesses des acteurs du système. Le « facteur humain » est celui qui est le plus difficile à évaluer, comprendre, et à maîtriser. Il est cause de biais et d'erreurs qui créent de l'incertitude, tant au niveau individuel que des groupes. Cette source est pernicieuse car souvent produite de bonne foi et donc susceptible de passer inaperçue. Elle peut être relative aux agents ou aux décideurs, porter sur des biais perceptifs, des erreurs de représentation, ou des influences ascendantes qui modifient la décision en fonction doptions prises à des niveaux inférieurs ou supérieurs, etc. (Sage 1981 ; Bushnell et al. 1988 ; Wiegmann et Scott 2006). Face au déluge d'information, différents filtres

8 Par exemple, le dépassement de la limite des microprocesseurs de 32-bits (ou nombre de Euler $=2^{\wedge \wedge} 31-1=$ 2.147.483.647) est connu comme ayant pour conséquences des erreurs informatiques potentiellement critiques, voire catastrophiques. 
physiques et contextuels peuvent réduire la quantité de celle qui est fournie au décideur. Bien que cette solution améliore la qualité de la conscience de la situation, et donc la qualité de la décision, Taylor et Snell (1988) ont montré que ce n'est pas le manque d'information de qualité qui est en cause dans les décisions inadéquates, mais le manque de capacité d'attention et de performances cognitives suffisantes du décideur. La solution proposée consiste à transférer le plus de charge de travail à des programmes d'Intelligence artificielle, d'augmentation et d'aide à la décision. Les données doivent donc être resynthétisées, fusionnées, en une information globalement intelligible et doivent être traitées pour générer des bases de connaissances contextuelles. L'enjeu de l'ingénierie humaine est justement de comprendre et maîtriser les causes de l'incertitude, en travaillant sur la robustesse des procédures, sur le partage représentationnel, sur la confiance relationnelle dans la maîtrise de l'erreur dans les systèmes complexes (Strauch 2007) et dans l'utilisation de systèmes cognitiques pour une cognition augmentée, une diminution de la complexité technologique et une contextualisation de la complexité (cf. figure 6).

La complexité dimensionnelle correspond au fait qu'un système de commande et contrôle est structuré de manière cybernétique, en une hiérarchie de sous-systèmes, dont chacun peut consister lui-même en un système cybernétique à part entière, lui-même composé de sous-systèmes, etc. (cf. figure $\mathrm{n}^{\circ} 2$ ). Le niveau cellulaire peut alors être considéré comme l'opérateur humain, lui-même étant un système cybernétique composé de systèmes internes, etc. Or, il existe une interaction mutuelle entre sous-systèmes concurrents et système englobant. L'ensemble des processus gérés ou produits aux différents niveaux génèrent eux-mêmes des interactions et régulations multiples, elles aussi en interaction entre elles. Le nombre de cas à considérer augmente de manière exponentielle, et cela alors que chaque interface peut générer des quantités considérables de messages différents. Si chacun d'entre eux peut être facilement traité et analysé de manière simple, le système fractal considéré dans son ensemble dépasse les capacités de compréhension et de représentation spontanée et les capacités de modélisation. Levis et Athans (op. cit.) parlent à ce propos de " malédiction de la dimensionnalité " en regrettant que l'ingénierie des systèmes traditionnels, où un problème est divisé en niveaux simples, ne puisse s'appliquer. Cooper (op. cit., 1994) remarque à ce propos une forme d'auto-similarité, ou de similarité entre les niveaux. Il suggère que le principe selon lequel chaque sous-système étant constitué de sous-sous-systèmes présentant une même structure cybernétique, et ainsi de suite, répond aux critères d'une structure complexe (Woodcock 1987). 


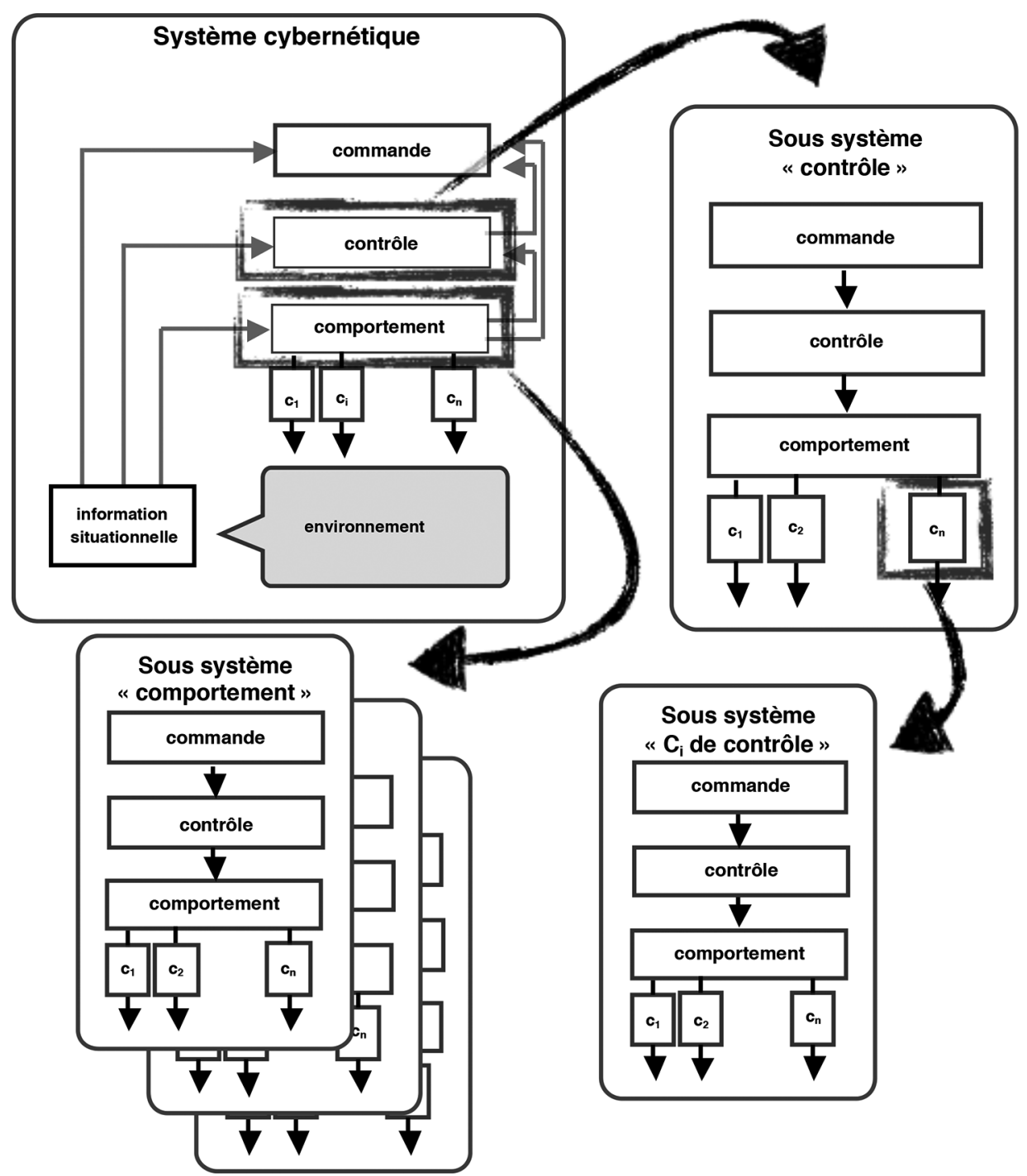

Figure 4 : modèle de décomposition d'éléments en sous-systèmes cybernétiques "Commande-Contrôle-Comportement », selon un principe d'autosimilarité. Modèle développé d'après la figure $n^{\circ} 1$

La complexité de calcul est celle que rencontrent les structures gérant de grands projets. Les dimensions de rapidité, de fiabilité et de puissance de calcul font en effet entrer le $\mathrm{C} 2$ et son immense quantité de données dans le monde du « big data ». Ce domaine qui connaît aujourd'hui un intérêt majeur des entreprises et des institutions présente les deux faces de son intérêt et de ses contraintes (Cointot et Eychenne 2014). Le big data est constitué de l'ensemble des données générées et recueillies en continu sans catégorisation a priori, et dont le volume croît de manière exponentielle. Elles génèrent de nouveaux 
usages, de nouvelles stratégies et de nouveaux enjeux pour ceux qui disposent des moyens informatiques et des algorithmes nécessaires à leur traitement. Renversement de la science expérimentale en une démarche prospective, le big data engage la société, ses entreprises et ses institutions dans l'exploitation des données qui deviennent l'une des énergies du monde économique et militaire de demain (Babinet 2015). Chacun s'accorde à penser que ceux qui maîtrisent l'exploitation du big data sont les détenteurs du techno-pouvoir de demain. Cette exploitation commence bien entendu par la maîtrise des capteurs mais également par le traitement des données ouvertes, de l' " open source ", et de l'information «broadcast " (Albert et Hayes 2006). La raison numérique est aujourd'hui circonscrite par une « anthropologie de l'exponentiel» (Sadin 2015) obéissant, pour les contenus, à la Loi que Gordon Moore (1965) avait formulée il y a un demi-siècle pour la puissance des composants numériques. Cette exploitation ouvre sur les algorithmes d'approximation du " data mining » et au débat sur l' " intelligence artificielle » (Delahaye 2002). Les techniques d'IA sont utilisées depuis longtemps dans différentes dimensions du C2 : aides de planification tactique (Akey et al. 1987), systèmes experts dans la gestion de bataille (Flynn et Senator 1987), aides à la surveillance (Goubert et Desjouis 1989), et plus généralement résolution de problèmes dans des environnements incertains (Andert 1992). L'IA et l'iconographie statistique sont à la base de la nécessaire réification des données. L'ensemble des informations données à chaque étage de décision est le reflet plus ou moins fidèle de certaines caractéristiques physiques du milieu, de la nature d'événements qui s'y produisent, et de données d'intelligence artificielle surajoutées. Il permet à chaque niveau d'avoir une « certaine conscience » du réel. Si les « data » sont censées en être des descriptions, elles sont interprétées et ne parviennent au commandeur que de manière spéculaire. Or, une donnée interprétée est plus ou moins discutable, et la démarche de $\mathrm{C} 2$ va alors être d'en fournir un inventaire avec des algorithmes de réponse immédiate ou différée en fonction de critères de choix que supportent de nécessaires métasystèmes d'intelligence artificielle.

Dans ce cadre, les limites des ordinateurs, comme outils de résolution de problèmes, peuvent être déterminées selon deux types (Taylor et Snell 1988). Les problèmes « calculables et programmables " sont ceux qui peuvent être résolus avec la technologie actuelle. C'est le cas des calculs par des algorithmes de tri ou ceux utilisant la transformée de Fourrier ou tous moyens de traitement du signal. En fait, ces problèmes ne constituent qu'une part des problèmes concernés par le $\mathrm{C} 2$. Les autres problèmes sont «programmables et non calculables». C'est ceux pour lesquels les algorithmes nécessitent des contraintes temporelles, de mémoire ou énergétiques trop grandes pour des solutions pratiques. La théorie de la complexité informatique se pose la question de savoir si la réponse à un problème peut être donnée très efficacement, efficacement ou au contraire être inatteignable en pratique ou 
en théorie (problèmes non déterministes par exemple). La qualification de la difficulté de niveaux intermédiaires entre les deux extrêmes se fonde sur une estimation des temps de calcul et des besoins en mémoire informatique. C'est donc à la fois une question de hardware et de software. Enfin, certains problèmes ne sont pas calculables. C'est principalement ceux qui concernent le facteur humain, l'impondérable et l'imprévisible, et qui font l'objet de procédures particulières pour peu qu'ils aient été imaginés par les concepteurs des programmes ou des procédures.

Cette théorie de la complexité établit des hiérarchies ou " classes de complexité ». Mais la complexité des opérations à réaliser a aussi des conséquences sur leur déroulement concret. Tel est le cas de la consommation d'énergie nécessaire à leur réalisation, tant pour alimenter les ordinateurs que pour les refroidir. Cette énergie peut varier considérablement suivant la performance des processus utilisés pour effectuer les calculs selon le « principe de Landauer " (Landauer 1961 ; Moore 2012). On sait par exemple que le « Power Usage Effectiveness » (PUE) qui est l'indicateur le plus utilisé pour comparer la consommation des centres de traitement de données (Avelar et al. 2012), dépasse de 13 à $20 \%$ la consommation directe du calcul, et cela pour les meilleures performances énergétiques des data centers. Cela veut dire que pour 1 kilowattheure d'électricité utilisée par les machines, il faut au moins 0,13 kilowattheure supplémentaire pour le stockage, la maintenance, le refroidissement, etc. Ne sont pas calculées les dépenses relatives à la sécurité physique et, paradoxalement, le coût homme nécessaire à la mise en œuvre, la maintenance et la sûreté du calcul. Nous pouvons raisonnablement faire l'hypothèse qu'il évolue également comme la puissance nécessaire et le besoin énergétique, c'est-à-dire de manière exponentielle.

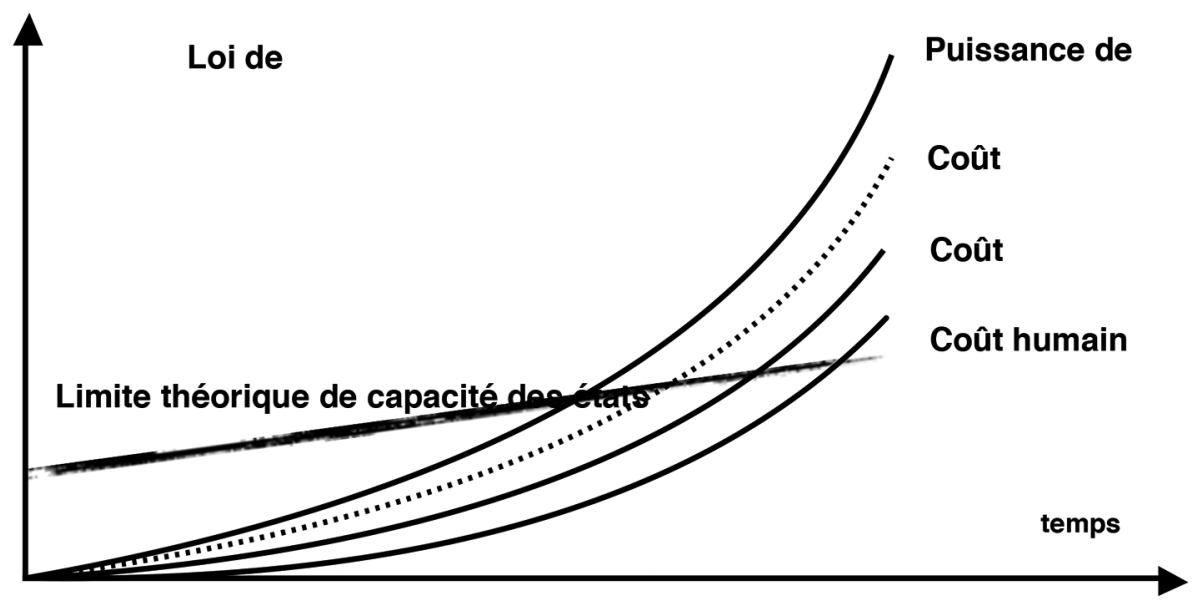

Figure 5 : évolution des coûts relative à l'évolution des puissances de calcul. La capacité théorique est donnée de manière arbitraire, à titre d'illustration du texte. 
Ces coûts cumulés d'investissement et de fonctionnement notamment énergétique, la maîtrise de l'empreinte carbone et l'investissement en spécialistes très qualifiés sont autant de facteurs qui éloignent progressivement le $\mathrm{C} 2$ des capacités matérielles, financières et humaines des sociétés de petite ou moyenne taille et de certaines nations qui doivent alors déléguer le commandement et contrôle de leurs missions.

\section{Accompagner la complexité}

L'un des principaux obstacles à la conception, la gestion et l'application du $\mathrm{C} 2$ est donc sa complexité. Historiquement issue de procédures accumulations évoluant à la fois au rythme des développements technologiques et des équilibres entre enjeux de sécurité et coûts réels, humains, écologiques et financiers, la déclinaison technique et procédurale du C4ISR rencontre aujourd'hui les limites de ces champs. La complexité des systèmes est une réalité, son évolution dynamique en est une autre, et le constat de sa prise en charge et de sa maîtrise par l'homme devient une nécessaire contrainte de l'usage des dispositifs technologiques de grande dimension. Le C2 n'y échappe pas et le dépassement de ces limites repose sur l'homme.

La place de l'homme dans la gestion de la complexité est aujourd'hui réexaminée par certains auteurs. Une certaine catégorie, dont les ergonomes de langue française considèrent que la complexité est une forme d'ennemi de l'homme au travail, une sorte de barrière ou de mur de verre, et qu'il fait la diminuer pour garder l'humanité au travail et surtout au futur technologique (Amalberti 1998). Cette perspective est basée sur les meilleures intentions et s'attache à la fois aux enjeux de protection du travailleur, à l'amélioration des conditions de travail et au bien-être dans son accomplissement.

Ce mouvement n'est pourtant pas exempt de défaut.Il érige en contrainte de facilité une certaine forme de lock-in dans la concurrence entre technologies évolutives, voire entre technologies rivales ou supplétives. Ce constat est à associer à la théorie de " dépendance au sentier " (Path dependancy; David 1985) selon laquelle il est souvent choisi des solutions moins efficaces car plus économiques à maintenir que développer et adopter un changement. Cette adaptation par maintien est ici à entendre au plan du coût humain du changement pour les agents concernés comme à celui du coût économique ou d'évolution procédurale ou doctrinale. On voit alors se développer à côté de cette ergonomie éclairée un certain mouvement qui, souhaitant maintenir l'homme au centre du progrès, érige petit à petit les barrières de protection contre des développements technologiques jugés trop rapides, trop stressants, voire réputés porter atteinte à la santé et au plein développement d'une catégorie théorique de travailleurs épanouis. Ce mouvement est de fait technophobe, quasi « neoludiste ». S'ils ne s'attachent pas réellement à « casser les machines » pour stopper la modernité, il n'en est pas loin dans l'esprit et, à notre avis, fédère déjà les exclus ou les perdants de demain. 
Certains autres auteurs, notamment inspirés du mouvement anglo-saxon du « human factor », considèrent au contraire qu'il faut s'adapter à la complexité et son inéluctable évolution dans les systèmes technologiques. La complexité n'est pas pour eux un mur, un handicap, ou une limite, mais plus une vague que l'on n'arrête pas et qu'il convient de négocier en privilégiant l'aptitude, la performance et l'aide matérielle ou stratégique pour surfer et avancer avec elle. Le rapport des systèmes technologiques complexes à l'homme est alors exprimé selon plusieurs dimensions. En premier lieu et de manière globale, plus les incertitudes, les ambiguités, et les inconnus sont importants, plus l'intervention humaine devient paradoxalement nécessaire, et plus la complexité augmente, plus le rôle de l'homme devient central. En second lieu, sur le registre matériel, plus le temps de la prise de décision diminue, plus la probabilité d'une décision humaine en dehors de procédures ou moyens d'aide artificielle augmente. Plus ces aides disponibles sont faillibles, plus souvent l'homme est appelé à combler les lacunes du système (Smith 2006).

La place de l'homme est différente dans ces deux approches. La première est celle d'un raisonnement performatif visant à orienter la recherche vers la maîtrise d'une complexité que l'on sait pourtant dynamique et auto créatrice de sa propre augmentation, la seconde réside dans l'analyse des moyens de formation et modélisation, d'analyse des données et d'aide à la décision, et de délégation à des processus d'intelligence artificielle d'augmentation. C'est cette voie qui est promue pour l'efficacité du commandement et contrôle.

La cognitique est l'étude des technologies cognitives et de leur rapport aux hommes. Cette science cognitive appliquée développe dans la perspective précédente trois moyens d'approche des relations et de l'intégration entre les hommes et les systèmes (Claverie 2005). La première consiste à simplifier les technologies et notamment les moyens et instruments de l'interface. Les méthodes concernent par exemple l'iconographie sémantique en réalité augmentée d'informations recomposées et utiles au moment et en contexte (Head up display et autres outils d'immersion et de réalité augmentée dans l'usine du futur ou en robotique chirurgicale, etc.). La seconde concerne l'augmentation des capacités humaines elles-mêmes, soit par des équipements numériques d'augmentation (exosquelettes, vision nocturne, etc.) mais aussi par tout procédé écologique d'influence positive (pharmacologie, stimulations et influences psychophysiologiques, etc.) afin de privilégier une insertion de l'hybridité anthropotechnique. La dernière repose plus sur des méthodes de pensée naturelle ou étayée, en facilitant la cognition par des procédures de contextualisation de la complexité, ou de formation à des stratégies efficaces et plus sûres dans la maîtrise de l'erreur ou dans celle de la gestion de l'affectivité et des procédures mentales. 


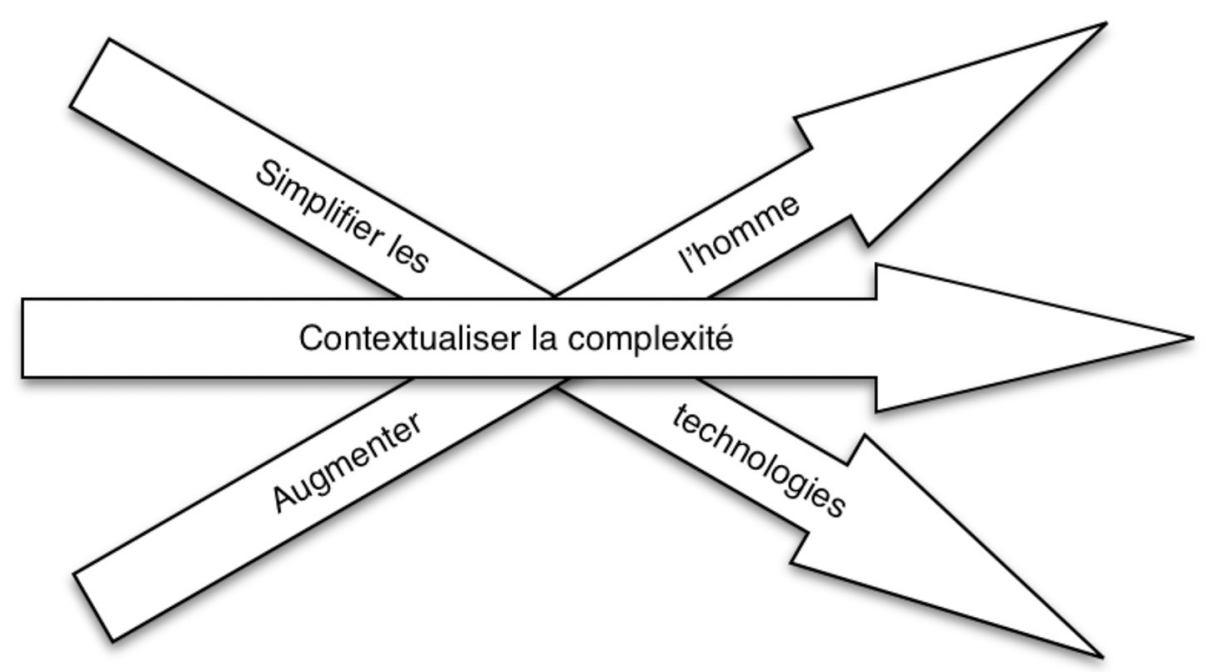

Figure 6 : les trois voies de la Cognitique (d'après Claverie 2005)

L'accompagnement de la complexité par l'homme immergé dans de grands systèmes technologiques tels que ceux que gère le $\mathrm{C} 2$ peut donc bénéficier de ces trois stratégies. Plus la technologie et ses interfaces se simplifient tout en présentant les informations pertinentes stratégiques, plus les incertitudes et les ambiguïtés sont minimisées. Nous préférons d'ailleurs au terme de simplification celui de clarification de l'information, notamment en référence au big data et aux outils de représentation de l'information. En découlent une diminution du taux nécessaire d'intervention humaine et un allégement des contraintes d'ergonomie de contrôle et d'ergonomie de correction. Ensuite, plus la contextualisation délimite la complexité du problème, plus la représentation adéquate de la réalité est favorisée. L'intervention de l'humain dans la boucle sera d'autant moins nécessaire pour des procédures de vérification, de contrôle et d'ajustement. Enfin, l'augmentation des performances dues à l'introduction de stratégies d'augmentation humaine et d'intelligence artificielle fournit un soutien rapide et performant pour libérer le temps pour la prise de décision. De cette manière, la probabilité d'une décision correcte augmente et l'opérateur sera moins souvent en situation d'ultime recours dans des situations critiques.

Le décalage de l'homme vis-à-vis de la complexité du système est source de difficultés croissantes qui limitent les degrés de liberté de progression de performance, engageant selon Amalberti (op. cit. 1998) une "rupture du paradigme des années 40 , et l'entrée dans un nouveau paradigme du presque tout automatique avec la suppression des opérateurs de proximité, et le transfert de contrôle vers très peu d'opérateurs centralisés et fortement assistés ». Une telle évolution n'est possible qu'avec un traitement « à 
cœur » du système en évitant de ne recourir qu'à un traitement de surface, une cosmétique d'interface, une contrainte de sentier de dépendance (path dependency) ou un simple aménagement des conditions de travail qui ne peuvent raisonnablement qu'être secondaires aux enjeux majeurs et critiques traités par le C2.

Dans ce cadre, la transformation des hiérarchies héritées de la guerre froide permet aujourd'hui d'aborder des stratégies d'agilité dans la Défense (Desclaux, op.cit. 2006), comme dans les grands ensembles industriels. La plasticité et l'évolution à la fois structurale, fonctionnelle et stratégique des conflits du XXI ${ }^{e}$ siècle sont des contraintes que le $\mathrm{C} 2$ doit assumer. Le concept de «power to the edge » est promu depuis quelques années par le DoD. Il consiste à valoriser les droits de décision (" peer-to-peer »), la dissémination de l'information ( "broad dissemination ») et l'interaction avec prise de décision au niveau le plus compétent et le mieux placé au moment opportun (" unconstrained interaction among the actors ") (Alberts et Hayes, op. cit., 2004, 2006). Ces dimensions s'accomplissent dans le cadre des objectifs généraux d'un commandement modulaire qui assume à la fois les directions, les contraintes de soutien et de sécurité et la formation des différents niveaux de $\mathrm{C} 2$, en implantant à la fois la clarification de l'information, sa contextualisation dans la complexité et l'augmentation des capacités humaines grâce au numérique.

\section{BIBLIOGRAPHIE}

AKEY M.,DUNKELBERGER K.,ERDMAN R. C., 1987, « Case studies in tactical decision support systems », in S. J. Andriole et J. L. Boyes (Eds.), Artificial Intelligence and National Defense, Washington (DC, USA) : AFCEA International Press (Armed Forces Communications and Electronics Association), 57-65.

ALBERTS D. S., HAYES R. E., 2004, Power to the Edge: Command, control, in the information age. Information Age Transformation Series. Washington (DC, USA) : US Department of Defense CCRP Publication Series.

ALBERTS D. S., HAYES R.E., 2006, Understanding Command and Control. Washington (DC, USA) : US Department of Defense CCRP Publication Series.

AMALBERTI R., 1998, «Les Facteurs Humains à l'aube de l'An 2000 ». Phoebus, 98: 5-12

ATHANS M., 1987, « Command and Control (C2) Theory: A Challenge to Control Science ». IEEE Transactions on Automatic Control, AC-32 : 4, 286-393.

ANDERT E.P., 1992, « Integrated knowledge-based system design and validation for solving problems in uncertain environments ", International Journal of Man-Machine Studies, 36: 2, 357-373.

AVELAR V., AZEVEDO D., FRENCH A. (Eds.), 2012, PUE : A Comprehensive Examination of the Metric, Beaverton (Oregon, USA) : The Green Grid Library.

BABINET G., 2015, Big data: Penser l'Homme et le Monde Autrement, Paris: Le Passeur Éditeur. 
BOYES, J. L., ANDRIOLE, S. (Eds.), 1987, Principles of Command and Control, Washington (DC, USA) : AFCEA International Press (Armed Forces Communications and Electronics Association).

BUILDER C. H., BANKES S. C., NORDIN R., 1999, Command Concepts a theory derived from the practicz of command and control. Santa Monica (Californie, USA): National Defense Research Institute, RAND.

BUSHNELL L. G., SERFATY D., KLEINMAN D. L., 1988, « Team information processing : a normative-descriptive approach », in S. E. Johnson et A.H. Levis (Eds.) Science of Command and Control: coping with uncertainty, Washington (DC, USA) : AFCEA International Press (Armed Forces Communications and Electronics Association), 62-72.

CLAVERIE B., 2005, Cognitique: science et pratique des relations à la machine à penser. Paris : L'Harmattan, collection « Cognition et formation ».

CLAVERIE B., DESCLAUX G., 2015, «La cybernétique: commande, contrôle et comportement dans la gestion des systèmes d'information et de communication». Hermes, 71: 72-79.

CLAUSEWITZ VON C., 1832, De la guerre (traduction De Vatry), Paris : Librairie militaire Baudoin, 1886, (traduction Neuens), Paris : Astrée, 2014.

COAKLEY T. P. (Ed.), 1991, C3I : Issues of Command and Control, Washington (DC, USA) : National Defense University Press.

COINTOT J.C., EYCHENNE Y., 2014, La Révolution Big Data : les Données au coeur de la Transformation de l'Entreprise, Paris : Dunod.

COOPER C., 1994, "Complexity in C3I systems », in D. Green et T. Bossomaier (Eds.) Complex systems: from biology to computation, Amsterdan (Pays Bas): IOS Press, 223-231.

COUfFIGNAL L., 1963, La Cybernétique, Paris : Presses Universitaires de France, collection «Que sais-je ? », n 638.

DAVID P. A.,1985, "Clio and the economics of QWERTY », American Economic Review, $75:$ 2, 332-337.

DAYAN M., 1966, Diary of the Sinai Campaign, London : Weidenfeld \& Nicolson.

DELAHAYE J. P., 2002, « La Loi de Moore », in J. P. Delahaye (Ed.) L'Intelligence du Calcul : de Gödel aux Ordinateurs Quantiques, Paris: Belin, 78-86.

DESCLAUX G., 2006, Transforming JC Lisbon : Tailored Task Organized DJTF Headquater Structure. Lisbonne (Portugal): NATO (white paper).

FLYNN J. P., SENATOR T. E., 1987, «DARPA naval battle management applications », in S. J. Andriole et J. L. Boyes (Eds.) Artificial Intelligence and National Defense, Washington (DC, USA): AFCEA International Press (Armed Forces Communications and Electronics Association), 66-82.

GOUBERT M., DESJOUIS M., 1989, « Using artificial intelligence for battlefield surveillance », Signal, 10: 69-73.

JOERGENSEN F. D., LARSEN P. G., STADTMUELLER J. M., 2005, «Untangling technology debates on information sharing and interoperability ». Military Communications Conference, 2005. MILCOM 2005. Atlantic City (New-Jersey, USA) : 17-20 octobre 2005. IEEE CECOM, 4, 2333-2338.

KOMETER M.W., 2007, Command in Air War : Centralized versus Decentralized 
Control of Combat Airpower, Montgomery (Alabama, USA): Air University Press.

LANDAUER R., 1961, "Irreversibility and heat generation in the computing process », IBM Journal of Research and Development, 5: 3, 183-191.

LARSEN P. G., 2006, «Coalition C2 Interoperability Challenges ». $11^{\text {th }}$. International Command and Control Research and Technology Symposium (ICCRTS-11), Cambridge (United Kingdom), 26 au 28 septembre 2006.

LE ROY F., 1997, De l'utilisation des doctrines militaires dans le domaine de l'entreprise, Annales des Mines, Gérer et Comprendre, 47: 89-96.

LEVIS A. H., ATHANS M., 1988, «The quest for a C3 theory: dreams and realities», in S.E. Johnson et A.H. Levis (Eds.) Science of Command and Control: coping with uncertainty, Washington (DC, USA): AFCEA International Press (Armed Forces Communications and Electronics Association), 4-9.

MOORE G. E., 1965, « Cramming More Components Onto Integrated Circuits », Electronics, $38:$ 4, 1-5.

MOORE S. K., 2012, “ Computing Power Limit Demonstrated », Spectrum, IEEE, 49: 5, 14-16.

OLMEDO E.,2010, «Complexity and chaos in organisations : complex management». International Journal of Complexity in Leadership and Management, 1: 1, 72-82.

RUDY M., 2006, " Pedagogical use of ELICIT for Leadership Training : Survey and Recommendations ». $16^{\text {th }}$ International Command E Control Research E Technology Symposium (ICCRTS-16). Québec (Canada), 21 au 23 juin 2011.

SAGE A. P., 1981, "Behavioral and organizational considerations in the design of information systems and processes for planning and decision support, IEEE Transactions on Systems, Man, and Cybernetics, SMC-11, 640-678.

SMITH E. A., 2006, Complexity, Networking, and Effects-based Approaches to Operations. Information Age Transformation Series. Washington (DC, USA): US Department of Defense CCRP Publication Series.

STEINBUCH K., 1957, "Informatik : Automatische Informationsverarbeitung ». SEG-Nachrichten (Berlin, Allemagne) Nr4: S, 171.

STRAUCH B., 2007, Investigagting Human Error: Incidents, Accidents, and Complex Systems. Aldershot (UK): Ashgate.

TAYLOR E. C., SNELL D. J., 1988, «Artificial intelligence in command and control», Signal, 4, 25-29.

TOSSEL C. C., SPLAWN J. C., DENNING T. D., BENNET W. R., 2008, "The Effectiveness of gaming Technology in Command and Control training ". 21th. American Psychology Association Annual Technical Symposium: Distributed Cognition: Teams, Technology, Family, and the Individual, Fairfax (Virginia USA), 6-7 mars 2008.

TOSSEL C. C., 2008, Collaborative Tool for C2 Team effectiveness Studies. Technical Report TR-08 1. Institute for Information Technology Applications, Washington (DC USA): US Air Force Academy.

YATES A. J., VERNIK R., MAHESWARAN N., ALLWRIGHT A. M., 1999, Systems Characterisation and Modelling Approaches for C3I (white paper), Canberra (Australie): Australia's Defence Science and Technology Organisation (ADSTO $\mathrm{ADoD})$.

WARDEN J., 1995, "Air Theory for the 21st century ", in B.R. Schneider, L. E. Grinter (Eds.) 
Battlefield of the Future : 21st Century Warfare Issues, Montgomery (Alabama, USA) : Air University Press, 103-123.

WEE C. H., LEE K. S., BAMBANG W. H., 1991, Sun Tzu: War and Management, Singapore: Addison-Wesley.

WOODCOCK A.E.R., 1987, "Catastrophes, Chaos, and C3I». IEEE 2nd International Conference on C2 information Technology (CIT) and Management Information Systems. Londres (UK): The Institution of Electrical Engineers. 3-5 mai 1987. Proceedings : IEEE Conference Pub, 275, 31-37.

WOODCOCK A.E.R., 1988, "Indications and warnings as an input to the C3 process ", in J. L. Boyes et S. J. Andriole (Eds.) Science of Command and Control: coping with uncertainty, Washington (DC, USA): AFCEA International Press (Armed Forces Communications and Electronics Association), 32-47.

WIEGMANN D. A., SCOTT A. S., 2006, A Human Error Approach to Aviation Accident Analysis. Aldershot (UK): Ashgate.

Collectif, 2006, ELICIT Report, Experiments in Command and Control within Edge Organizations, Command and Control Research Program, Washington (DC, USA) : US Department of Defense CCRP Publication Series.

Collectif, 2015, C4ISR\&Networks. Managing massive data (white paper). Washington (DC - USA): Air Force Cyber Initiatives, 2015

Collectif - Joint Chiefs Of Staff Dod, 2008, Department Of Defense Dictionary Of Military And Associated Terms: Joint Publication 1-02, Washington (DC, USA): Government Printing Office (GPO), GPO Bookstore.

Résumé : Le «Command and Control» ou « $\mathrm{C} 2$ » (traduit parfois par « commandement et conduite ») correspond au volet théorique d'un ensemble de moyens et méthodes (C4ISR) de gestion de grands systèmes complexes humains engagés habituellement dans des opérations dont l'absence de maîtrise peut avoir des conséquences hautement regrettables, voire catastrophiques. Le présent article décrit ce volet théorique en relation avec sa déclinaison pratique et instrumentale, et montre comment il s'insère dans un mouvement de régulation de la complexité tout en étant lui-même générateur de cette complexité. L'analyse s'inscrit dans le registre cybernétique et explore, notamment pour le domaine militaire, les registres de cette complexité et le statut du $\mathrm{C} 2$ comme système de systèmes.

Mots-clés: commandement et conduite, commandement, contrôle, complexité, cybernétique, management complexe, militaire, stratégie, système de systèmes.

Summary: The "Command and Control» or «C2» is the theoretical part of a set of means and methods (CAISR) for managing large human complex systems usually engaged in operations whose lack of effective management can have highly undesirable or even disastrous consequences. This study investigates the theory that supports C4ISR practice as well as its role in the complexity of regulating movement while being himself generator of its complexity. The present analysis belongs to cybernetics and explores mostly military applications the ranges of this complexity and the status of $C 2$ as a system of systems.

Keywords: command and control, complexity, cybernetics, management of complexity, military, strategy, system of systems. 